\title{
The Association with Quantitative Response to Attention-Deficit/Hyperactivity Disorder Medication of the Previously Identified Neurodevelopmental Network Genes
}

\author{
Yuanxin Zhong, MS, ${ }^{1, *}$ Binrang Yang, $\mathrm{PhD},{ }^{2, *}$ Yi Su, MD, PhD, ${ }^{1}$ Ying Qian, MD, $\mathrm{PhD},{ }^{1}$ \\ Qingjiu Cao, MD, PhD, ${ }^{1}$ Suhua Chang, PhD, ${ }^{1}$ Yufeng Wang, MD, PhD, and Li Yang, MD, PhD ${ }^{1}$
}

\begin{abstract}
Objective: A recent pharmacoimaging study suggested that methylphenidate (MPH) and atomoxetine (ATX) might have common mechanisms for the treatment of attention-deficit/hyperactivity disorder (ADHD). Previous pharmacogenetic studies have by and large only involved genes in neurotransmitter systems, which accounted for very small variances. Therefore, this study aimed to investigate whether the neurodevelopmental genes identified in a prior ADHD etiology Genome-Wide Association Study (GWAS) could predict patients' responses to MPH and ATX, given the aforementioned mechanisms of action. Methods: For our sample of 241 patients with ADHD, we assessed the change in the ADHD rating scale (ADHD-RS) total symptom scores from baseline to the end of the 12th week of treatment with either MPH or ATX. We performed association analyses at the genetic single-marker, gene-based, set-based, and GWAS-based polygenic levels.

Results: In our analyses, neither single nucleotide polymorphism (SNP) nor gene-level analyses yielded significant markers associated with the change in the ADHD-RS score after multiple comparison correction. The polygenic risk score model, which was based on SNPs associated with ADHD etiology at a threshold of $p \leq 0.0001$ in a recent Han Chinese GWAS, predicted symptomatic improvement with ADHD medication $\left(p=0.018, R^{2}=0.023\right)$.

Conclusion: Our results provide new evidence for a small influence of neurodevelopmental genes on the efficacy of medications for ADHD.
\end{abstract}

Keywords: ADHD, pharmacogenetics, neurodevelopmental genes, polygenic risk score

\section{Introduction}

A TTENTION-DEFICIT/HYPERACTIVITY DISORDER (ADHD) is a common behavioral disorder with an onset in childhood. The most recent meta-analyses showed that the prevalence is $7.2 \%$ worldwide (Thomas et al. 2015) and 6.26\% in China (Wang et al. 2017). Many follow-up studies revealed that the symptoms and impairment induced by ADHD might extend into adulthood (Cadman et al. 2016). It not only impaired academic difficulties but also increased the risk for antisocial behavior and substance abuse, which burdened both the family and society (Tarver et al. 2015).
A recent population investigation of 760,000 school-aged children in Scotland showed a higher rate of hospitalization and injury in children with ADHD, which suggested that the fate of children with ADHD was worse than that of their peers not only in education but also in health (Curry et al. 2017; Fleming et al. 2017; Ostergaard et al. 2017; Chen et al. 2019). These findings indicated that ADHD required effective intervention.

Pharmacological treatment is the most prevalent therapy for ADHD. In China, the most commonly used drugs are preparations of methylphenidate (MPH) and atomoxetine (ATX). Both drugs significantly improve the symptoms and cognitive function of

\footnotetext{
${ }^{1}$ Peking University Sixth Hospital (Institute of Mental Health), National Clinical Research Center for Mental Disorders \& Key Laboratory of Mental Health (Peking University), Ministry of Health, Beijing, China.

${ }^{2}$ Shenzhen Children's Hospital, Shenzhen, China.

*Both these authors contributed equally to this work.

Funding: This work was supported by grants from the National Natural Science Foundation of China (81471381 to L.Y.), the Sanming Project of Medicine in Shenzhen "The ADHD research group from Peking University Sixth Hospital" (SZSM201612036), the National Natural Science Foundation of China (81701348 to Y.S., 81471382 to Q.C., and 81671358, 8171101223, and 81873803 to L.Y.), the National Key R\&D Program of China (2016YFC1306103), and the Clinical Medicine Plus X-Young Scholars Project of Peking University, the Fundamental Research Funds for the Central Universities.
} 
ADHD (Newcorn et al. 2008; Schulz et al. 2012; Yang et al. 2012; Su et al. 2016), which suggests that they may act on some common pathways. For example, in recent neuroimaging studies, both MPH and ATX were shown to change the activation of the prefrontal and central motor cortex (Schulz et al. 2012; Smith et al. 2013; Cubillo et al. 2014). It was also well documented that both MPH and ATX act by blocking the cortical norepinephrine transporter (Florin et al. 1994; Bymaster et al. 2002; Yang et al. 2004). All this evidence suggested common therapeutic mechanisms for stimulant and nonstimulant medications.

The response to medication is different across individuals with ADHD, with a $\sim 75 \%$ response rate with MPH and only a $50 \%$ response rate with ATX. The response varied regarding the optimal effect, doses, duration of effect, and tolerability. It was reported that some factors, including symptom severity at referral, age, perinatal complications, attention test score, intelligence quotient (IQ), clumsiness, emotional disorders, learning disorders, and response to single-dose MPH, could predict medication response (Buitelaar et al. 1995; Treuer et al. 2014; Johnston et al. 2015; Silberstein et al. 2017), although there remained a lack of consistent results. To date, there has been no index that could reliably predict the drug response. Clinicians have to use a "trial and error" step-by-step process to determine the best medication. This may lead to a delay in achieving the therapeutic effect and to unnecessary adverse effects.

It has long been acknowledged that the genetic constitution plays an important role in the body's response to external substances. However, in the past 20 years, there has not been much progress in understanding the pharmacogenetics of ADHD medication. Most studies involved genes of the dopamine and norepinephrine systems based on the pharmacological mechanisms of these drugs. In a recent meta-analysis, only variants in SLC6A2, ADRA2A, SLC6A3, $D R D 4$, and $C O M T$ were replicated associated with MPH efficacy, and the odds ratios for each variant were low. A multivariate predictor with sufficient accuracy is necessary for use in clinical practice (Myer et al. 2018).

Genome-wide association studies revealed many neurodevelopmental genes to be responsible for ADHD. The evidence includes the first GWAS performed by Neale et al. (2008), which found that some genes involved in cell adhesion, neuron migration, neurogenesis, synapse plasticity, as well as transcription factors were potentially associated with ADHD. Franke et al. (2012) drew a gene network related to neurite outgrowth for ADHD using bioinformatic analyses. Yang et al. (2013) investigated common and rare variants in Han Chinese. Although no genome-wide significant locus was identified, the potential associated genes included neurodevelopmental genes that were enriched in two Gene Ontology terms, namely, neuronal projection and synapses.

Therefore, this study aimed to further investigate the pharmacogenetics based on new findings about the etiology of ADHD. It explored the association between variants of neurodevelopmental genes and medication responses of two major drugs used in the treatment of ADHD. The hypothesis was that gene variants associated with the etiology of ADHD also predict medication efficacy. Identifying susceptibility genes for drug response might help to establish a predictive model before prescription, which could lead to individualizing precision medicine.

\section{Materials and Methods}

\section{Subjects}

Children and adolescents who met the ADHD criteria of the Diagnostic and Statistical Manual of Mental Disorders, 4th ed.
(DSM-IV), were recruited from the Child and Adolescent Psychiatric Outpatient Department of Peking University Sixth Hospital. The sample comprised two populations: one treated by immediaterelease MPH before 2010 when intermediate-released (IR)-MPH was the only choice on the Chinese market and the other treated by osmotic release oral system methylphenidate (OROS MPH) and ATX in a randomized parallel study (Yang et al. 2012).

The diagnosis was initially made by a child psychiatrist and then validated by a semistructured interview with the parents using Barkley's Clinical Diagnostic Interview Scale (Barkley 1998). This scale was based on DSM-IV criteria. It included questions about the 18 items regarding ADHD symptoms, age of onset, impairment of function, and exclusion criteria. We used this scale in our previous pharmacogenetic studies (Yang et al. 2004, 2013). All patients met the following symptom severity thresholds: a total score of investigator-rated ADHD rating scale (ADHD-RS)-IV of no $<25$ for boys or 22 for girls or a subtype-corresponding subscale score equal to or more than 12 (DuPaul et al. 1998; Yang et al. 2007).

Most of the subjects were medication naive when recruited. Only $13(7.6 \%)$ in the MPH group and $4(5 \%)$ in the ATX group had a treatment history with MPH but had stopped for at least 1 week. No subject had previous ATX treatment. All patients and their parents were Han Chinese. The exclusion criteria were as follows: (1) allergy to MPH or ATX, (2) combined treatment with other psychotropic drugs or nondrug intervention for ADHD, and (3) noncompliance with the blood collection procedure. The sample was composed of 241 children with ADHD, which included 202 boys and 39 girls. The study was approved by the Peking University Sixth Hospital Institutional Review Board. Parents signed written informed consent. For the children and adolescents, oral assent was acquired. No incentive was provided to the study participants, except for necessary transport reimbursement for each visit in the second sample.

\section{Treatment}

The subjects received open-label treatment with either intermediate-release MPH, OROS MPH, or ATX for 8-12 weeks. The dose was titrated to the optimal dosage over 3-4 weeks. Then, the dose was maintained for at least 8 weeks. Patients experiencing side effects at any stage of titration could be maintained at that dose for $1-2$ weeks. The final average dosage was $0.45 \pm 0.11 \mathrm{mg} /(\mathrm{kg} \cdot$ day $)$ for IR-MPH, $0.69 \pm 0.19 \mathrm{mg} /(\mathrm{kg} \cdot$ day $)$ for OROS MPH, and $1.1 \pm 0.2 \mathrm{mg} /(\mathrm{kg} \cdot$ day $)$ for ATX. The total course of treatment was no more than 12 weeks. Following a baseline assessment, the treatment response was assessed at the end of the 1st, 2nd, 4th, 8th, and 12th weeks. Medication compliance was assessed by directly asking the parent at every visit. Patients who missed a whole or a partial dose for 3 consecutive days or for 10 days in total were defined as noncompliant and dropped out of the trial.

We analyzed the reasons for drop-out and found that the primary reason was adverse events, while the second most common reason was lack of efficacy (OROS MPH, 7.6\%, and ATX, 16.1\%). We also compared the difference in clinical characteristics between completers and drop-outs, which revealed no statistically significant differences (Su et al. 2016).

\section{Genotyping}

The samples were genotyped using the Affymetrix6.0 array from CapitalBio Ltd. (Beijing, China) using the standard Affymetrix protocol. The Affymetrix6.0 array included 906,600 single nucleotide polymorphism (SNP) probes. The SNP genotypes were called 
with BIRDSEED v2. A total of 241 cases passed the first-stage sample control with call rates $>98 \%$, no first- or second-degree relative relationships, and genders consistent with site reports. For the inclusion of SNPs, we required a call rate $>95 \%$, minor allele frequency $>1 \%$, and Hardy-Weinberg equilibrium $p$-value $>10^{-6}$. We extracted those SNPs in the developmental genes suggested in our previous GWAS (Yang et al. 2013) for candidate gene-based analyses (Table 1).

\section{Statistical analysis}

Outcome variable and covariates. The primary efficacy measure was the investigator-rated ADHD-RS (DuPaul et al. 1998). It was rated based on both parent and teacher reports. The ADHD-RS consisted of 18 items corresponding to DSM criteria for ADHD. The total symptom scores as well as the inattention and hyperactive-impulsive subscale scores were used to evaluate the core symptoms of ADHD. This scale was translated into Chinese. The validity and reliability of the Chinese version were demonstrated by Su et al. (2015). In the present study, we used the change in the ADHD-RS score from baseline to the end of the 12th treatment week as the outcome. Since the baseline symptoms and changes in scores between drugs were not significantly different, we did not add the treatment type as a covariate to avoid overcorrection.

Genetic single-marker analysis. For each SNP, we performed an association analysis between the SNP and the change in ADHD-RS score using PLINK 1.07 (Purcell et al. 2007). We then conducted a linear regression analysis to adjust for the association $p$-value using age, baseline symptom severity, and IQ as covariates (Kim et al. 2017).

Gene-based analysis. Compared with a single-marker analysis, a gene-based analysis combines all SNPs within a gene. Gene-based tests were conducted to determine gene-level associations by summing the total effect of a set of SNPs harbored in a gene using the SKAT test by Efficient and Parallelizable Association Container Toolbox (EPACTS). In brief, these tests explored associations on a per-gene basis taking into account the $p$-value of all the SNPs within each gene, adjusting for the covariates of sex, IQ, baseline symptom severity, and the top 4 eigenvectors of the principal component analysis calculated for SNPs in low linkage disequilibrium $\left(r^{2}=0.1\right)$ by EIGENSTRAT 4.2 software (Patterson et al. 2006; Price et al. 2006). We used Bonferroni for multiple comparison correction. Since there were 7532 genes included in the analysis, the significant threshold was $0.05 / 7532=6.64 \mathrm{e}-6$.

Set-based analysis. To further analyze the effect of all the neurodevelopmental genes suggested in our previous Han Chinese ADHD GWAS (Yang et al. 2013), we combined all the SNPs in the genes into a larger set and performed a set-based analysis. This was a strategy to test the pathway association of functionally related genes (Medina et al. 2009). We used the SKAT test by EPACTS. We included the same quality control standards and covariates in this analysis.

The polygenic risk score. Polygenic risk score (PRS) is a quantitative measure of the total contribution of common genetic variants to a trait and is calculated as a sum of multiple SNP alleles associated with a trait, typically weighted by effect sizes estimated from a GWAS study (Guo et al. 2018). As a measure of the genetic susceptibility to the treatment response, PRS was based on data from our ADHD genome-wide association study (Yang et al. 2013).

We calculated the PRS based on the statistic derived from the discovery sample, which comprised 769 ADHD cases and 897 controls, leaving out the cases of this study for an independent target sample. A total of 759 cases and 896 controls remained after quality control (described in the Genotyping section). The association analysis was conducted using an additive logistic regression model in PLINK 1.07 (Purcell et al. 2007). In accordance with other studies, including PRSs (Yuan et al. 2018), we selected SNPs associated with ADHD at a predefined $p$-value threshold of 0.0001 , which was also the threshold we used to find the neurodevelopmental gene network in the ADHD GWAS (Yang et al. 2013). We identified a total of 540,821 SNPs. The changes in scores on the ADHD-RS were regressed on PRSs by PRSice (Euesden et al. 2015). The same covariates were included as in the above analyses. The significance of the regression results was corrected by a permutation test with 10,000 replicates.

Genetic analyses combining the two medications and separate for MPH and ATX were performed at each level mentioned above.

\section{Results}

There were 68, 93, and 80 patients in the intermediate-release MPH group, OROS MPH group, and ATX group, respectively (Table 2).

Table 1. Neurodevelopmental Genes Included in the Gene-Based Analyses

\begin{tabular}{|c|c|c|c|c|c|c|c|c|c|c|c|}
\hline \multicolumn{4}{|c|}{ Synapse } & \multicolumn{4}{|c|}{ Axon } & \multicolumn{4}{|c|}{ Synapse and axon } \\
\hline Gene & Chr & Start & End & Gene & $C h r$ & Start & End & Gene & Chr & Start & End \\
\hline PCDH15 & 10 & $55,562,531$ & $57,387,702$ & ITGAI & 5 & $52,083,730$ & $52,255,040$ & CNTN2 & 1 & $205,012,325$ & $205,047,627$ \\
\hline$P R K C Q$ & 10 & $6,469,105$ & $6,622,263$ & KLHL1 & 13 & $70,274,725$ & $70,682,625$ & GRIK4 & 11 & $120,382,468$ & $120,859,613$ \\
\hline$C A L D 1$ & 7 & $134,429,003$ & $134,655,480$ & CNTNAP2 & 7 & $145,813,453$ & $148,118,090$ & GRM3 & 7 & $86,273,230$ & $86,494,200$ \\
\hline KCTD16 & 5 & $143,550,396$ & $143,865,249$ & $N F A S C$ & 1 & $204,797,779$ & $204,991,950$ & $N R X N 1$ & 2 & $50,145,643$ & $51,259,674$ \\
\hline$S V 2 C$ & 5 & $75,378,997$ & $75,649,764$ & SCN10A & 3 & $38,738,293$ & $38,835,501$ & GRM7 & 3 & $6,811,688$ & $7,783,218$ \\
\hline SYT10 & 12 & $33,527,173$ & $33,592,754$ & SCN11A & 3 & $38,887,260$ & $38,992,052$ & & & & \\
\hline EPHA7 & 6 & $93,949,738$ & $94,129,300$ & SLC38A1 & 12 & $46,576,838$ & $46,663,800$ & & & & \\
\hline LRFN2 & 6 & $40,359,325$ & $40,555,204$ & & & & & & & & \\
\hline PSD3 & 8 & $18,384,811$ & $18,942,240$ & & & & & & & & \\
\hline PICK1 & 22 & $38,452,318$ & $38,471,708$ & & & & & & & & \\
\hline
\end{tabular}

Chr, chromosome. 
Table 2. Baseline Characteristic and Medication Response of the 241 Patients

\begin{tabular}{|c|c|c|c|c|c|}
\hline Characteristic & $\begin{array}{c}\text { Intermediate-released MPH }(\mathrm{n}=68) \\
\text { Mean }(S D)\end{array}$ & $\begin{array}{c}\text { OROS MPH }(\mathrm{n}=93) \\
\text { Mean }(S D)\end{array}$ & $\begin{array}{c}\operatorname{ATX}(\mathrm{n}=80) \\
\text { Mean }(S D)\end{array}$ & $F / \chi^{2}$ & $\mathrm{p}$ \\
\hline Age & $9.1(2.1)$ & $9.2(2.1)$ & $9.5(2.3)$ & 0.67 & 0.72 \\
\hline IQ & $102.5(15.3)$ & $104.0(15.1)$ & $105.0(14.8)$ & 0.63 & 0.53 \\
\hline ADHD-RS baseline score & $33.5(9.4)$ & $32.9(8.8)$ & $32.8(8.2)$ & 0.12 & 0.89 \\
\hline \multirow[t]{2}{*}{ ADHD-RS decreased score } & $11.1(12.1)$ & $14.4(9.6)$ & $13.4(8.2)$ & 3.60 & 0.17 \\
\hline & $\mathrm{n}(\%)$ & $\mathrm{n}(\%)$ & $\mathrm{n}(\%)$ & & \\
\hline Sex & & & & 0.19 & 0.91 \\
\hline Male & $57(83.8)$ & $79(84.9)$ & $66(82.5)$ & & \\
\hline Female & $11(16.2)$ & $14(15.1)$ & $14(17.5)$ & & \\
\hline ADHD subtype & & & & 2.80 & 0.25 \\
\hline Inattentive & $21(30.9)$ & $28(30.1)$ & $33(41.3)$ & & \\
\hline Combined comorbidity & $47(69.1)$ & $65(69.9)$ & $47(58.8)$ & & \\
\hline ODD & $21(30.9)$ & $32(34.4)$ & $29(36.3)$ & 0.48 & 0.79 \\
\hline
\end{tabular}

ADHD, attention-deficit/hyperactivity disorder; ADHD-RS, ADHD Rating Scale; ATX, atomoxetine; IQ, intelligence quotient; MPH, methylphenidate; ODD, oppositional defiant disorder; OROS MPH, osmotic release oral system methylphenidate; SD, standard deviation.

\section{Genetic single-marker analyses}

Although we observed some nominal signals, for example, rs 13225250 in $C N T N A P 2$ (uncorrected $p$-value $=0.0011$ ), rs 12475081 in NRXN1 (uncorrected $p$-value $=0.0037$ ), and rs17384265 in PSD3 (uncorrected $p$-value $=0.0042$ ), none passed the multi-comparison correction for the association with the change in the ADHD-RS score (Table 3). Only rs1 1004071 in $P C D H 15$ yielded nominal significance in the separate analyses for MPH (uncorrected $p$-value $=0.049$ ) and ATX (uncorrected $p$-value $=0.042$ ).

\section{Gene-based analyses}

We focused on candidate neurodevelopmental genes. The genebased analyses did not reveal significant results that survived correction for multiple comparisons. Table 4 shows the associations between neurodevelopmental genes and quantitative response to ADHD medication treatment. Gene names, the number of SNPs,

Table 3. The Association with Quantitative Response to Attention-Deficit/Hyperactivity Disorder Medication for the Top Single Nucleotide POLYMORPHISMS FROM THE NeURODEVELOPMENTAL NeTWORK GENES

\begin{tabular}{|c|c|c|c|c|c|}
\hline \multirow[b]{2}{*}{ Gene } & \multirow[b]{2}{*}{$C h r$} & \multicolumn{4}{|c|}{ Quantitative response } \\
\hline & & SNPs & $A 1$ & $\begin{array}{l}\text { Uncorrected } \\
\text { p-value }\end{array}$ & $\begin{array}{c}\text { Corrected } \\
\text { p-value }\end{array}$ \\
\hline CNTNAP2 & 7 & rs13225250 & $\mathrm{C}$ & 0.020 & 1.00 \\
\hline KLHL1 & 13 & rs7985990 & G & 0.020 & 1.00 \\
\hline NFASC & 1 & rs11240315 & $\mathrm{C}$ & 0.011 & 0.78 \\
\hline SCN10A & 3 & rs7635221 & $\mathrm{T}$ & 0.018 & 0.24 \\
\hline SLC $38 A 1$ & 12 & rs17096738 & $\mathrm{T}$ & 0.019 & 0.44 \\
\hline NRXN1 & 2 & rs 12475081 & $\mathrm{C}$ & 0.0037 & 1.00 \\
\hline GRM7 & 3 & rs3749380 & $\mathrm{T}$ & 0.038 & 1.00 \\
\hline PSD3 & 8 & rs17384265 & G & 0.0042 & 1.00 \\
\hline CALD1 & 7 & rs2218988 & $\mathrm{C}$ & 0.038 & 1.00 \\
\hline PCDH15 & 10 & rs11004071 & A & 0.017 & 1.00 \\
\hline$S V 2 C$ & 5 & rs2937715 & $\mathrm{T}$ & 0.015 & 1.00 \\
\hline GRIK4 & 11 & rs6589850 & G & 0.025 & 1.00 \\
\hline
\end{tabular}

$\mathrm{Chr}$, chromosome; SNP, single nucleotide polymorphism. and uncorrected $p$-values are provided. None of the genes showed a significant association in combined or separate analyses for the two medications.

\section{Set-based analyses \\ of the neurodevelopmental pathway}

To further detect the combined effect of all the neurodevelopmental genes suggested in the Han Chinese ADHD GWAS, we

Table 4. Gene-Based Analysis of Associations Between Neurodevelopmental Genes and Quantitative RESPONSES to ATtENTION-DEFICIT/HyPERACTIVITY DisORDER MEDICATION

\begin{tabular}{lrccc}
\hline & & \multicolumn{3}{c}{$\mathrm{p}$} \\
\cline { 3 - 5 } Gene & No. of SNPs & Combined & MPH & ATX \\
\hline KLHL1 & 90 & 0.19 & 0.81 & 0.88 \\
CALD1 & 18 & 0.22 & - & - \\
CNTNAP2 & 700 & 0.35 & 0.0080 & 0.21 \\
ITGA1 & 75 & 0.35 & 0.25 & 1.00 \\
SCN10A & 34 & 0.38 & 0.12 & 0.64 \\
GRIK4 & 168 & 0.45 & 0.23 & 0.25 \\
CNTN2 & 18 & 0.50 & 0.82 & 0.36 \\
NFASC & 73 & 0.59 & - & - \\
SYT10 & 32 & 0.60 & - & - \\
NRXN1 & 320 & 0.77 & - & - \\
PRKCQ & 60 & 0.78 & 0.51 & 0.36 \\
PCDH15 & 409 & 0.80 & 0.37 & 0.51 \\
SV2C & 119 & 0.80 & 0.63 & 0.73 \\
GRM7 & 372 & 0.87 & - & - \\
EPHA7 & 33 & 0.89 & 0.15 & 0.91 \\
PSD3 & 247 & 0.91 & 0.92 & 0.86 \\
GRM3 & 39 & 1.00 & 0.030 & 0.64 \\
KCTD16 & 59 & 1.00 & - & - \\
LRFN2 & 100 & 1.00 & 1.00 & 0.0069 \\
SCN11A & 20 & - & 0.68 & 0.26 \\
SLC38A1 & 14 & - & 0.63 & 0.57 \\
\hline
\end{tabular}

Combined, combined sample of MPH and ATX groups.

- No association was found with quantitative response.

ATX, atomoxetine; MPH, methylphenidate; SNP, single nucleotide polymorphism. 
Table 5. Associations Between the Neurodevelopmental Pathway Gene Set and Quantitative Response to Attention-Deficit/ Hyperactivity Disorder Drugs

\begin{tabular}{lccc}
\hline & Combined & MPH & ATX \\
\hline No. of SNPs & 3069 & 1670 & 1664 \\
$p$ & 0.31 & 0.69 & 0.078 \\
\hline
\end{tabular}

Combined, combined sample of MPH and ATX groups.

ATX, atomoxetine; MPH, methylphenidate; SNP, single nucleotide polymorphism.

conducted set-based analyses. We observed that the association between the set and the changes in scores on the ADHD-RS did not achieve significance in either combined or separate analyses (Table 5).

\section{Polygenic risk score}

We investigated the predictive ability of a model constructed from SNPs linked to ADHD etiology at the threshold of 0.0001 in the Han Chinese ADHD GWAS (Yang et al., 2013). The variance explained $\left(R^{2}\right)$ and regression coefficients of the PRS and their corresponding $p$-values were calculated using an additive regression model. A significant $p$-value of 0.018 was obtained for the change in the ADHD-RS score (Table 6). A significant result was also observed in the MPH-treated group $\left(R^{2}=0.026, p=0.038\right)$ but not in the ATX group $\left(R^{2}=0.015, p=0.28\right)$.

\section{Discussion}

In the past two decades, psychiatric pharmacogenetic studies have heavily focused on the genes in the pharmacological pathway, that is, neurotransmitter-related genes. Recent psychiatric genomic studies revealed many neurodevelopmental genes as susceptibility genes for psychiatric disorders. Some studies found that these neurodevelopmental genes also influenced medication response. For example, NRXN1 was found to be involved in antidepressant activity as well as drug responses in patients with schizophrenia (Jenkins et al. 2014), while CNTNAP2 was found to be involved in both the susceptibility and long-term treatment outcomes of bipolar disorder (Fabbri and Serretti 2016).

The present study was based on the hypothesis that neurodevelopmental gene variants associated with the etiology of ADHD also predicted medication response. We performed analyses at the single-variant, gene-based, set-based, and polygenic levels.

Both SNP and gene-level analyses yielded no significant results associated with the change in the ADHD-RS score after multiple

Table 6. Polygenic Modeling Using Single Nucleotide Polymorphisms from the Discovery SAMPle to Predict IMPROVEMENT IN ATtENTION-Deficit/HyPERACTIVITY Disorder Rating Scale After Treatment

\begin{tabular}{lcccl}
\hline Sample & $P T$ & No. of SNPs & $\mathrm{R}^{2}$ & \multicolumn{1}{c}{$\mathrm{p}$} \\
\hline Combined & 0.0001 & 35 & 0.023 & 0.018 \\
MPH & & & 0.026 & 0.038 \\
ATX & & & 0.015 & 0.28
\end{tabular}

Combined, combined sample of MPH and ATX groups.

ATX, atomoxetine; MPH, methylphenidate; PT, predefined threshold; SNP, single nucleotide polymorphism. comparison corrections, which might be due to the small effect of each SNP or gene on the response variance. However, some potential associations in the results were interesting. Some SNPs were found to be nominally associated with symptom improvement, for example, rs17384265 in PSD3 and rs13225250 in CNTNAP2. These genes are involved in cell adhesion (CNTNAP2) and neurite/dendrite outgrowth (PSD3).

Combining a number of SNPs that show modest associations with a trait such as treatment response might explain a significant portion of the variance (Yuan et al. 2018). To obtain a larger effect size, we combined the genes in the neurodevelopmental pathway (Yang et al. 2013) to perform the set-based analysis. However, we did not find a significant result for the association with symptom improvement, although this might be due to the small sample size.

In addition, we used a polygenic analysis method, using the Han Chinese ADHD GWAS study as the discovery sample (Yang et al. 2013). It provided statistically significant evidence for the association between the PRS and the change in the ADHD-RS score. The model constructed from SNPs linked to ADHD etiology at the threshold of 0.0001 in the Han Chinese ADHD GWAS succeeded in the prediction of symptom improvement, accounting for $2.28 \%$ of the variance in outcomes. In stratified analyses, a significant result was also found in MPH-treated patients, although not in ATX-treated patients, possibly due to the small sample size for the ATX group or to different mechanisms of action for MPH and ATX (Tzavara et al. 2006; Shang et al. 2016).

The significant prediction by PRS was in agreement with some studies on antipsychotic medications (GENDEP Investigators et al. 2013). A small predictive ability was found for the polygenic risk score calculated in a meta-analysis of GWAS of depression in the GENDEP-MARS studies in STAR*D (GENDEP Investigators et al. 2013), in which $\sim 1.2 \%$ of the variance accounted for the antidepressant response in major depressive disorder. However, inconsistent results were also found. One study reported no evidence for PRS predictive ability in response to obsessive compulsive disorder (Alemany-Navarro et al. 2019), although the findings require replication in larger sample sizes (Guo et al. 2018).

Since there was a difference between the results from the setbased analysis and the PRS model, we further annotated the SNPs that passed the threshold in the analysis of PRS according to their position on the Genome Reference Consortium February 2009 build of the human genome (GRCh37/hg19). From these results, we found 35 SNPs included in the analyses after clustering, and the majority of the SNPs were located in intergenic regions or the introns of neurodevelopment-related genes, which were likely regulatory elements involved in complex regulation. For example, rs4074793 in ITGA1, rs4936537 in GRIK4, rs2685159 in CTNNA2, rs7094454 in CTNNA3, rs12445560 in CPNE7, rs2423037 in $R A S S F 2$, and rs 2327046 in PLCB1. The SNPs that we combined in the set-based analysis were all SNPs located in the selected genes. Therefore, in addition to the small sample size, different numbers and locations of SNPs representing the genes for set analyses and PRS might lead to inconsistency.

To the best of our knowledge, this was the first study to use a PRS to predict the response to pharmacological treatment in ADHD. There were some limitations in this study, including the open-label, nonrandomized study design and lack of intention-totreat analyses. In addition, the lower final daily doses for MPH administered, which may be linked to the lower-than-expected remission rate observed for OROS MPH, may have limited the ability to detect a therapeutic response in these cohorts. Furthermore, although we detected that the PRS explained a significant amount of 
variance in the outcome, the effect was small and perhaps clinically insignificant. Therefore, replication of these results in a larger sample with an optimized study design is necessary to determine the relevance of the findings reported.

\section{Conclusion}

The study suggested, to a large extent, that the pharmacological and etiological pathways of ADHD might be distinct from each other, as genetic predictors of the disorder explain only a small portion of the variance in medication response. Indeed, current drugs only relieve symptoms but do not cure disease. Thus, our results indirectly supported the need to develop new drugs that target the ADHD etiology and not just the symptoms.

\section{Clinical Significance}

The current study suggests the different mechanisms underlying ADHD and treatment response, respectively. The predictive model for treatment response needs to be improved.

\section{Acknowledgment}

The authors thank all patients for participating in this study.

\section{Disclosures}

No competing financial interests exist.

\section{References}

Alemany-Navarro M, Costas J, Real E, Segalas C, Bertolin S, Domenech L, Rabionet R, Carracedo A, Menchon JM, Alonso P: Do polygenic risk and stressful life events predict pharmacological treatment response in obsessive compulsive disorder? A geneenvironment interaction approach. Transl Psychiatry 9:70, 2019.

Barkley RA: Attention-Deficit Hyperactivity Disorder: A Clinical Workbook, 2nd ed. New York: Guilford, 1998, p. 628.

Buitelaar JK, Van der Gaag RJ, Swaab-Barneveld H, Kuiper M: Prediction of clinical response to methylphenidate in children with attention-deficit hyperactivity disorder. J Am Acad Child Adolesc Psychiatry 34:1025-1032, 1995.

Bymaster FP, Katner JS, Nelson DL, Hemrick-Luecke SK, Threlkeld PG, Heiligenstein JH, Morin SM, Gehlert DR, Perry KW: Atomoxetine increases extracellular levels of norepinephrine and dopamine in prefrontal cortex of rat: A potential mechanism for efficacy in attention deficit/hyperactivity disorder. Neuropsychopharmacology 27:699-711, 2002.

Cadman T, Findon J, Eklund H, Hayward H, Howley D, Cheung C, Kuntsi J, Glaser K, Murphy D, Asherson P: Six-year follow-up study of combined type ADHD from childhood to young adulthood: Predictors of functional impairment and comorbid symptoms. Eur Psychiatry 35:47-54, 2016.

Chen Q, Hartman CA, Kuja-Halkola R, Faraone SV, Almqvist C, Larsson H: Attention-deficit/hyperactivity disorder and clinically diagnosed obesity in adolescence and young adulthood: A registerbased study in Sweden. Psychol Med 49:1841-1849, 2019.

Cubillo A, Smith AB, Barrett N, Giampietro V, Brammer MJ, Simmons A, Rubia K: Shared and drug-specific effects of atomoxetine and methylphenidate on inhibitory brain dysfunction in medicationnaive ADHD boys. Cereb Cortex 24:174-185, 2014.

Curry AE, Metzger KB, Pfeiffer MR, Elliott MR, Winston FK, Power TJ: Motor vehicle crash risk among adolescents and young adults with attention-deficit/hyperactivity disorder. JAMA Pediatr 171: 756-763, 2017.
DuPaul GJ, Ervin RA, Hook CL, McGoey KE: Peer tutoring for children with attention deficit hyperactivity disorder: Effects on classroom behavior and academic performance. J Appl Behav Anal 31:579-592, 1998.

Euesden J, Lewis CM, O'Reilly PF: PRSice: Polygenic Risk Score software. Bioinformatics 31:1466-1468, 2015.

Fabbri C, Serretti A: Genetics of long-term treatment outcome in bipolar disorder. Prog Neuropsychopharmacol Biol Psychiatry 65: 17-24, 2016.

Fleming M, Fitton CA, Steiner MFC, McLay JS, Clark D, King A, Mackay DF, Pell JP: Educational and health outcomes of children treated for attention-deficit/hyperactivity disorder. JAMA Pediatr 171:e170691, 2017.

Florin SM, Kuczenski R, Segal DS: Regional extracellular norepinephrine responses to amphetamine and cocaine and effects of clonidine pretreatment. Brain Res 654:53-62, 1994.

Franke B, Faraone SV, Asherson P, Buitelaar J, Bau CH, RamosQuiroga JA, Mick E, Grevet EH, Johansson S, Haavik J, Lesch KP, Cormand B, Reif A: The genetics of attention deficit/hyperactivity disorder in adults, a review. Mol Psychiatry 17:960-987, 2012.

GENDEP Investigators; MARS Investigators; STAR*D Investigators: Common genetic variation and antidepressant efficacy in major depressive disorder: A meta-analysis of three genome-wide pharmacogenetic studies. Am J Psychiatry 170:207-217, 2013.

Guo W, Machado-Vieira R, Mathew S, Murrough JW, Charney DS, Grunebaum M, Oquendo MA, Kadriu B, Akula N, Henter I, Yuan P, Merikangas K, Drevets W, Furey M, Mann JJ, McMahon FJ, Zarate CA, Jr., Shugart YY: Exploratory genome-wide association analysis of response to ketamine and a polygenic analysis of response to scopolamine in depression. Transl Psychiatry 8:280, 2018.

Jenkins A, Apud JA, Zhang F, Decot H, Weinberger DR, Law AJ: Identification of candidate single-nucleotide polymorphisms in NRXN1 related to antipsychotic treatment response in patients with schizophrenia. Neuropsychopharmacology 39:2170-2178, 2014.

Johnston BA, Coghill D, Matthews K, Steele JD: Predicting methylphenidate response in attention deficit hyperactivity disorder: A preliminary study. J Psychopharmacol 29:24-30, 2015.

Kim JI, Kim JW, Park JE, Park S, Hong SB, Han DH, Cheong JH, Choi JW, Lee S, Kim BN: Association of the GRIN2B rs2284411 polymorphism with methylphenidate response in attention-deficit/ hyperactivity disorder. J Psychopharmacol 31:1070-1077, 2017.

Medina I, Montaner D, Bonifaci N, Pujana MA, Carbonell J, Tarraga J, Al-Shahrour F, Dopazo J: Gene set-based analysis of polymorphisms: finding pathways or biological processes associated to traits in genome-wide association studies. Nucleic Acids Res 37: W340-344, 2009.

Myer NM, Boland JR, Faraone SV: Pharmacogenetics predictors of methylphenidate efficacy in childhood ADHD. Mol Psychiatry 23: 1929-1936, 2018.

Neale BM, Lasky-Su J, Anney R, Franke B, Zhou K, Maller JB, Vasquez AA, Asherson P, Chen W, Banaschewski T, Buitelaar J, Ebstein R, Gill M, Miranda A, Oades RD, Roeyers H, Rothenberger A, Sergeant J, Steinhausen HC, Sonuga-Barke E, Mulas F, Taylor E, Laird N, Lange C, Daly M, Faraone SV: Genome-wide association scan of attention deficit hyperactivity disorder. Am J Med Genet B Neuropsychiatr Genet 147b:1337-1344, 2008.

Newcorn JH, Kratochvil CJ, Allen AJ, Casat CD, Ruff DD, Moore RJ, Michelson D: Atomoxetine and osmotically released methylphenidate for the treatment of attention deficit hyperactivity disorder: Acute comparison and differential response. Am J Psychiatry 165: 721-730, 2008.

Ostergaard SD, Dalsgaard S, Faraone SV, Munk-Olsen T, Laursen TM: Teenage parenthood and birth rates for individuals with and 
without attention-deficit/hyperactivity disorder: A nationwide cohort study. J Am Acad Child Adolesc Psychiatry 56:578.e3-584.e3, 2017.

Patterson N, Price AL, Reich D: Population structure and eigenanalysis. PLoS Genet 2:e190, 2006.

Price AL, Patterson NJ, Plenge RM, Weinblatt ME, Shadick NA, Reich D: Principal components analysis corrects for stratification in genome-wide association studies. Nat Genet 38:904-909, 2006.

Purcell S, Neale B, Todd-Brown K, Thomas L, Ferreira MA, Bender D, Maller J, Sklar P, de Bakker PI, Daly MJ, Sham PC: PLINK: A tool set for whole-genome association and population-based linkage analyses. Am J Hum Genet 81:559-575, 2007.

Reynolds GP, Yao Z, Zhang X, Sun J, Zhang Z: Pharmacogenetics of treatment in first-episode schizophrenia: D3 and 5-HT2C receptor polymorphisms separately associate with positive and negative symptom response. Eur Neuropsychopharmacol 15:143-151, 2005.

Schulz KP, Fan J, Bedard AC, Clerkin SM, Ivanov I, Tang CY, Halperin JM, Newcorn JH: Common and unique therapeutic mechanisms of stimulant and nonstimulant treatments for attentiondeficit/hyperactivity disorder. Arch Gen Psychiatry 69:952-961, 2012.

Shang CY, Yan CG, Lin HY, Tseng WY, Castellanos FX, Gau SS: Differential effects of methylphenidate and atomoxetine on intrinsic brain activity in children with attention deficit hyperactivity disorder. Psychol Med 46:3173-3185, 2016.

Silberstein RB, Levy F, Pipingas A, Farrow M: First-dose methylphenidate-induced changes in brain functional connectivity are correlated with 3-month attention-deficit/hyperactivity disorder symptom response. Biol Psychiatry 82:679-686, 2017.

Smith A, Cubillo A, Barrett N, Giampietro V, Simmons A, Brammer M, Rubia K: Neurofunctional effects of methylphenidate and atomoxetine in boys with attention-deficit/hyperactivity disorder during time discrimination. Biol Psychiatry 74:615-622, 2013.

Su Y, Yang L, Stein MA, Cao Q, Wang Y: Osmotic release oral system methylphenidate versus atomoxetine for the treatment of attention-deficit/hyperactivity disorder in chinese youth: 8-Week comparative efficacy and 1-year follow-up. J Child Adolesc Psychopharmacol 26:362-371, 2016.

Su YE, Wang H, Geng YG, Sun L, Du YS, Fan F, Su LY: Parent ratings of ADHD symptoms in chinese urban schoolchildren: Assessment with the Chinese ADHD rating scale-IV: Home version. J Atten Disord 19:1022-1033, 2015.

Tarver J, Daley D, Sayal K: Beyond symptom control for attentiondeficit hyperactivity disorder (ADHD): What can parents do to improve outcomes? Child Care Health Dev 41:1-14, 2015.

Thomas R, Sanders S, Doust J, Beller E, Glasziou P: Prevalence of attention-deficit/hyperactivity disorder: A systematic review and meta-analysis. Pediatrics 135:e994-e1001, 2015.
Treuer T, Feng Q, Desaiah D, Altin M, Wu S, El-Shafei A, Serebryakova E, Gado M, Faries D: Predictors of pharmacological treatment outcomes with atomoxetine or methylphenidate in patients with attention-deficit/hyperactivity disorder from China, Egypt, Lebanon, Russian Federation, Taiwan, and United Arab Emirates. Int J Clin Pract 68:1152-1160, 2014.

Tzavara ET, Bymaster FP, Overshiner CD, Davis RJ, Perry KW, Wolff M, McKinzie DL, Witkin JM, Nomikos GG: Procholinergic and memory enhancing properties of the selective norepinephrine uptake inhibitor atomoxetine. Mol Psychiatry 11:187-195, 2006.

Wang T, Liu K, Li Z, Xu Y, Liu Y, Shi W, Chen L: Prevalence of attention deficit/hyperactivity disorder among children and adolescents in China: A systematic review and meta-analysis. BMC Psychiatry 17:32, 2017.

Yang L, Cao Q, Shuai L, Li H, Chan RC, Wang Y: Comparative study of OROS-MPH and atomoxetine on executive function improvement in ADHD: A randomized controlled trial. Int J Neuropsychopharmacol 15:15-26, 2012.

Yang L, Neale BM, Liu L, Lee SH, Wray NR, Ji N, Li H, Qian Q, Wang D, Li J, Faraone SV, Wang Y: Polygenic transmission and complex neuro developmental network for attention deficit hyperactivity disorder: Genome-wide association study of both common and rare variants. Am J Med Genet B Neuropsychiatr Genet 162b: 419-430, 2013.

Yang L, Wang YF: Factors affecting therapeutic effect of ADHD treated by optimal dose of methylphenidate [in Chinese]. Beijing Da Xue Xue Bao Yi Xue Ban 39:284-288, 2007.

Yang L, Wang YF, Li J, Faraone SV: Association of norepinephrine transporter gene with methylphenidate response. J Am Acad Child Adolesc Psychiatry 43:1154-1158, 2004.

Yang L, Wang YF, Qian QJ, Biederman J, Faraone SV: DSM-IV subtypes of ADHD in a Chinese outpatient sample. J Am Acad Child Adolesc Psychiatry 43:248-250, 2004.

Yuan H, Wang Q, Liu Y, Yang W, He Y, Gusella JF, Song J, Shen Y: A rare exonic NRXN3 deletion segregating with neurodevelopmental and neuropsychiatric conditions in a three-generation Chinese family. Am J Med Genet B Neuropsychiatr Genet 177:589-595, 2018.

Address correspondence to: Li Yang, MD, PhD

Peking University Sixth Hospital (Institute of Mental Health) National Clinical Research Center for Mental Disorders \& Key Laboratory of Mental Health (Peking University) Ministry of Health Beijing 100191

China

E-mail: yangli_pkuimh@bjmu.edu.cn 